\title{
Eye metastasis in breast cancer: case report and review of literature
}

\author{
Ereny Samwel Poles Saad, HebatAllah Mahmoud Bakri, Amal Rayan, Dina Barakat and Mariam Mohsen Khalel
}

Department of Clinical Oncology, Faculty of Medicine, Assiut University, Kornish Al Ibrahimeya, Asyut Second, Assiut Governorate, Assiut 71515, Egypt

\section{Abstract}

The paradigm of breast cancer management has been revolutionised, resulting in prolonged survival that echoes an increasing incidence of metastasis in uncommon sites. With orbital metastases - despite being rare - the incidence scales up to $13 \%$ of breast cancer cases with no specific management guidelines. We report a case of a 31-yearold luminal B breast cancer patient who initially presented with T2N2M0 disease and received six cycles of adjuvant chemotherapy (5-Fluorouracil (5-FU) $600 \mathrm{mg} / \mathrm{m}^{2} \mathrm{IV}$, Doxorubicin $60 \mathrm{mg} / \mathrm{m}^{2} \mathrm{IV}$, Cyclophosphamide $600 \mathrm{mg} / \mathrm{m}^{2} \mathrm{IV}$ ), followed by radiotherapy (RTH) and adjuvant Tamoxifen. Two years later, the patient experienced successive bone metastasis, so she received several lines of endocrine therapy as Fulvestrant and aromatase inhibitors in combination with luteinizing hormone-releasing hormone (LHRH) analogues. Later on, she presented with right eye ptosis and magnetic resonance imaging (MRI) showed a soft tissue mass in the superior and lateral rectus muscles. The patient received six cycles of chemotherapy with no improvement. Further disease progression occurred 3 months later, so the patient received palliative RTH resulting in no response. One month later, the patient was deceased, secondary to progressive disease. With the rising incidence of ocular metastasis due to breast cancer, oncologists should be aware of symptoms and use the proper diagnostic modalities. Here we provide a literature review on similar cases and discuss possible treatment modalities for those patients. The main concern is to evaluate the need for chemotherapy in such cases in the presence of highly effective endocrinal treatment.

Keywords: breast cancer, eye metastases, treatment, chemotherapy, radiotherapy

\section{Introduction}

Eye metastases are a rare event in cancer patients with breast cancer being the most common primary site (28.5\%-58.8\%) [1]. The rising incidence of eye metastasis of breast cancer origin can be attributed to the recent advances in the systemic treatment of breast cancer which has resulted in prolonged survival of breast cancer patients in addition to the improvements in diagnostic modalities [2, 3]. In most cases, eye metastasis occurs along with systemic progression of previously diagnosed breast cancer; however, $25 \%$ of diagnosed eye metastases are detected in patients with de novo breast cancer as an initial presentation [4].
Correspondence to: Ereny Samwel Poles Saad Email: Erenysamwel@aun.edu.eg

ecancer 2022, 16:1353

https://doi.org/10.3332/ecancer.2022.1353

Published: $10 / 02 / 2022$

Received: 24/11/2021

Publication costs for this article were supported by ecancer (UK Charity number 1176307).

Copyright: (c) the authors; licensee ecancermedicalscience. This is an Open Access article distributed under the terms of the Creative Commons Attribution License (http:// creativecommons.org/licenses/by/4.0), which permits unrestricted use, distribution, and reproduction in any medium, provided the original work is properly cited. 
The aim of this literature review is to demonstrate different treatment modalities in cases described in the literature to help to evaluate best treatment options in addition to explaining our local experience with a case of breast cancer with eye metastasis in terms of the challenges in treatment based on the limited resources available.

\section{Patient information, clinical finding, diagnostic assessment}

We report a case of a 31-year-old patient who was diagnosed with T2N2M0 Estrogen Receptor(ER)/Progesterone (PgR) positive/HER-2/ neu negative, $\mathrm{Ki}-67>30 \%$ right breast cancer. She was treated with modified radical mastectomy followed by adjuvant chemotherapy with six cycles of FAC regimen (5-Fluorouracil (5-FU) $600 \mathrm{mg} / \mathrm{m}^{2} \mathrm{IV}$, Doxorubicin $60 \mathrm{mg} / \mathrm{m}^{2} \mathrm{IV}$, Cyclophosphamide $600 \mathrm{mg} / \mathrm{m}^{2} \mathrm{IV}$ ) every 21 days, radiotherapy (RTH) and adjuvant hormonal treatment with Tamoxifen for 2 years. The patient presented with severe back pain and the bone scan showed multiple bone metastases while multi-slice computed tomography (MSCT) of the chest and pelvis-abdomen were insignificant so she was shifted to luteinizing hormone-releasing hormone (LHRH) analogues in combination with Fulvestrant and palliative RTH. Eleven months later, the patient experienced successive bone progression and thereafter was shifted to aromatase inhibitors. Six months later, on November 2019 ( 4 years after the initial diagnosis), the patient presented with right eye ptosis, and the magnetic resonance imaging (MRI) of the brain and orbit showed a soft tissue mass in the superior and lateral rectus muscles (Figure 1) with no evidence of visceral metastasis on further metastatic work up except for multiple bone lesions.

\section{Therapeutic intervention and outcome of treatment}

After a multidisciplinary discussion, she started a combination chemotherapy of cisplatin and gemcitabine with a stationary course followed by Anastrozole. Three months later, the patient experienced further progression of her symptoms, in the form of ulceration, severe pain and pus discharge. The patient received palliative RTH at the dose of $30 \mathrm{~Gy}$ in ten fractions with no response (Figures 2 and 3). The patient was deceased 1 month later upon the deterioration of the general condition and further disease progression.

\section{Discussion}

Metastatic carcinoma of the eye is an uncommon clinical situation, and the most prevalent primary tumour is breast carcinoma which accounts for $28.5 \%-58.8 \%$ of all orbital metastases [3] followed by lung cancer (24\%) and skin melanoma (14\%) [1]. The increased incidence of eye metastasis of breast cancer follows the advances in diagnostic modalities and the prolonged survival of breast cancer patients. MRI remains the gold standard diagnostic imaging modality [5].

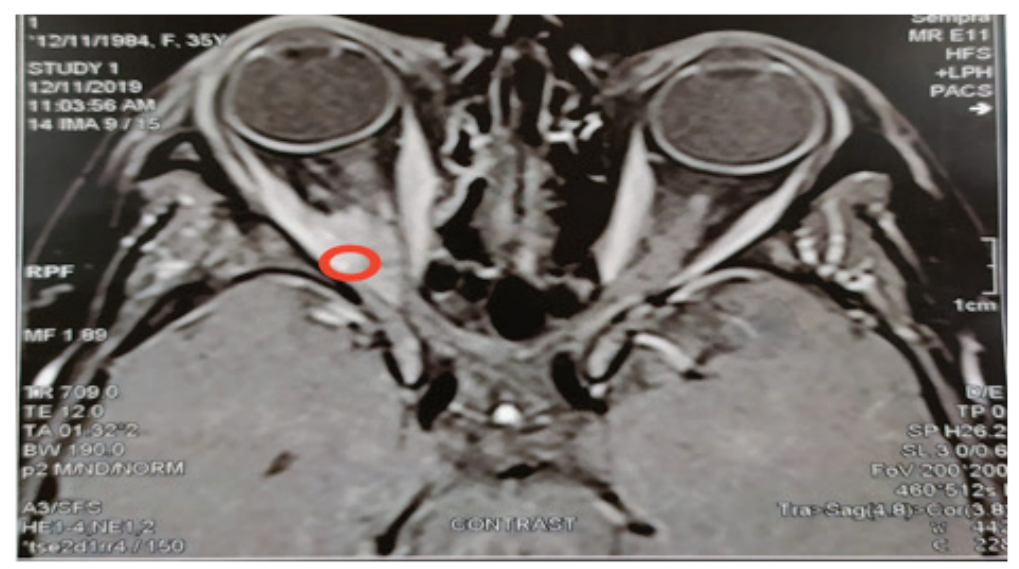

Figure 1. MRI brain at dignosis: T1 with contrast shows right superior rectal muscle thickening about 14 mm associated with slight proptosis of the right eye. 


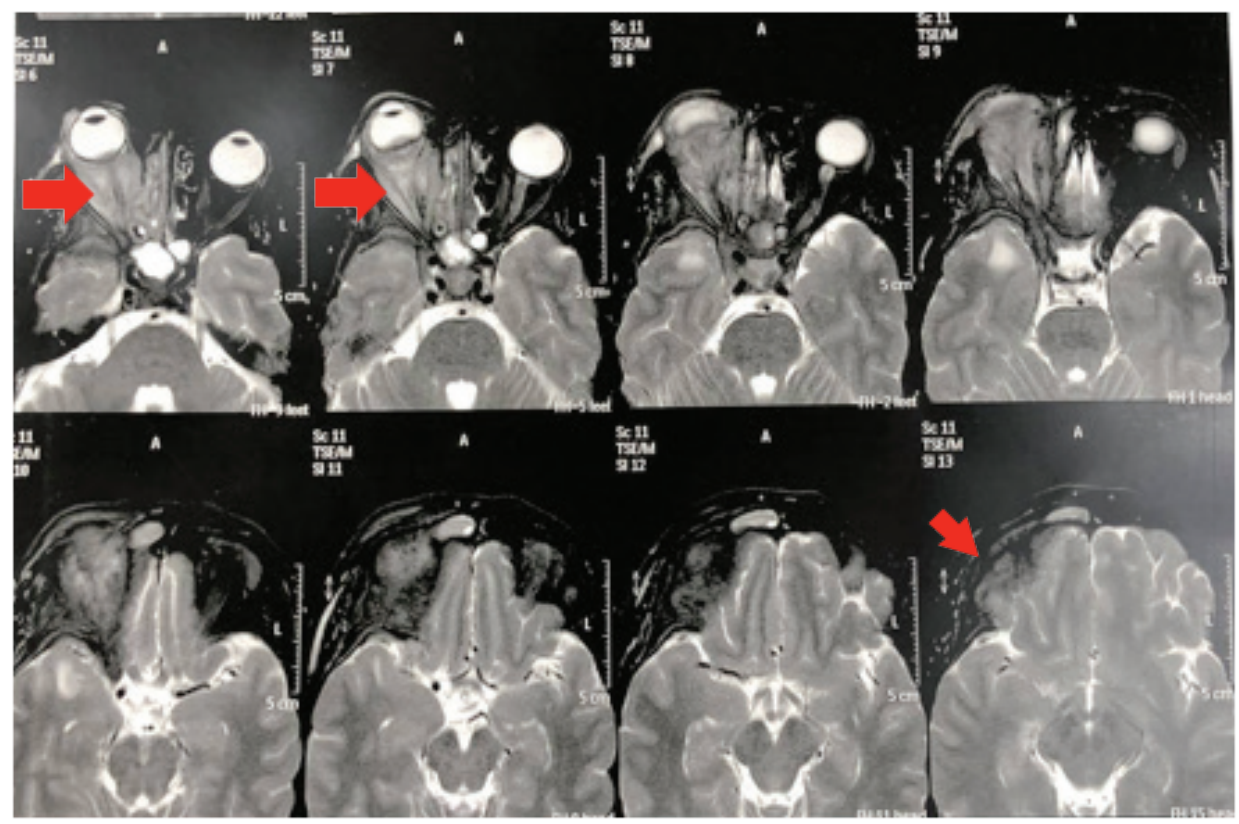

Figure 2. MRI brain and orbit showed significant increase in the previously described right recti muscles thickness with heterogeneous post-contrast enhancement. There is subsequent proptosis and posterior ocular coat mass lesion just above the optic disc with significant enhancement of all posterior ocular coats. There is extra-orbital spread, and intra-cranial extra-axial leptomeningeal enhancement at the right frontotemporal region.
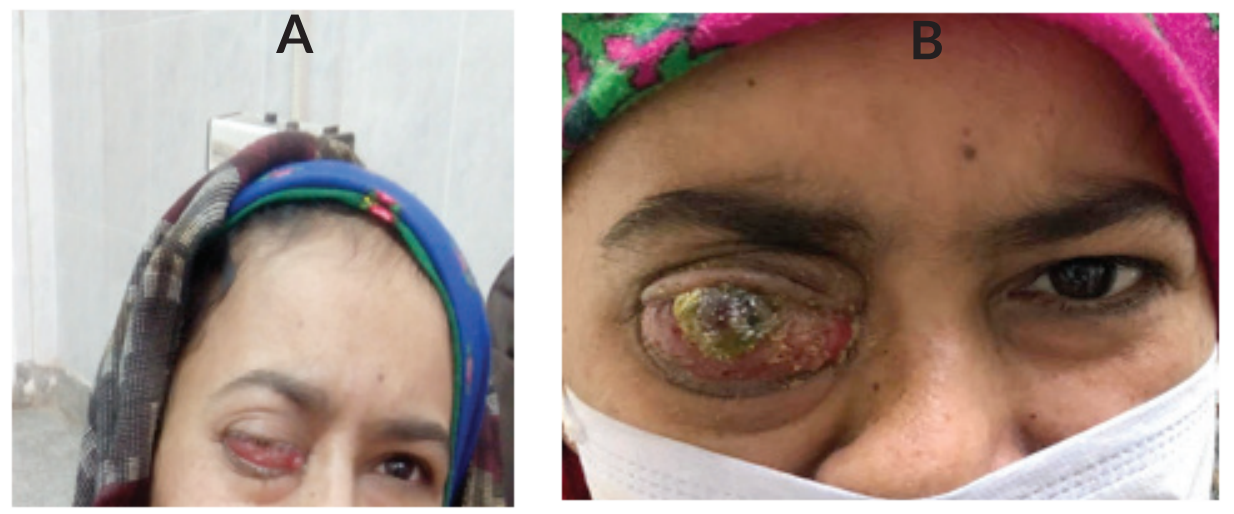

Figure 3. The presentation of the patient after the end of chemotherapy (a) and on progression after the radiotherapy (b).

A PubMed and Scopus search including English language only was performed using the Med search words 'breast cancer', 'eye metastasis' and/or 'orbital metastasis' until January 2021. The literature search revealed 53 records, of which 13 were included in the review and 40 in the quantitative analysis (Figure 4) with 94 cases collectively which were included in the analysis (Supplementary Table 1).

Eye metastases secondary breast cancer may be presented at any time point of the course of the disease, 38 (40.4\%) of cases included in the analysis presented with eye metastases as the initial presentation of breast cancer, while 56 (59.5\%) developed eye metastases either as 
the only site of metastasis or as a part of the systemic progression of previously diagnosed breast cancer. In those patients, the time interval between the diagnosis of breast cancer and the development of eye metastasis when reported had a wide range from 1 month [6] up to 25 years [7], and 13 cases developed eye metastasis within 5 years of being diagnosed with early breast cancer. In another review, Freedman et al [8] reviewed the charts of 112 patients (141 eyes) and showed that the average time was approximately 4 years (1,266 days) from the breast cancer diagnosis to the occurrence of metastasis to the eye and orbit.

Evaluation of the most common sites of the eye to be affected with metastasis of breast cancer was available for 66 cases as there was an overlap of data presented in one report as shown in Figure 5 [9]. There is controversy about the affinity of the breast cancer cell to specific tissue types within the eye; despite the extra-ocular muscles are rare to be affected [10] - based on the fact that the constant movement of muscles would prevent lodging of neoplastic cells [11] - but they were involved in one-third of the reviewed cases. Orbital involvement with annexes like the lacrimal gland was the second common site followed by the infiltrative mixed lesions that could affect more than one definitive structure. The uveal tract involvement was infrequent when compared with previous reports [12, 13].

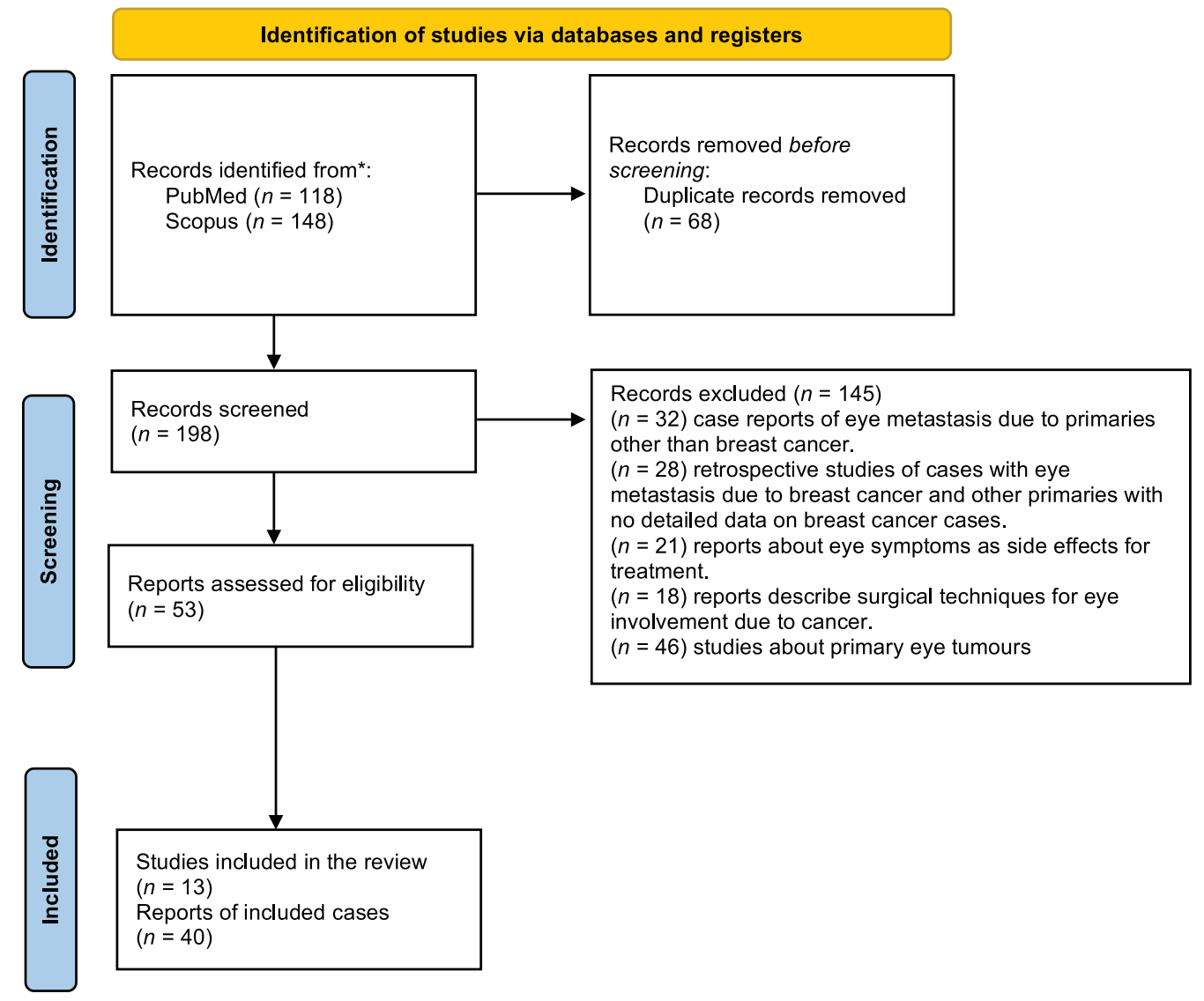

Figure 4. PRISMA flow diagram. From: Page MJ, McKenzie JE, Bossuyt PM, Boutron I, Hoffmann TC, Mulrow CD, et al. The PRISMA 2020 statement: an updated guideline for reporting systematic reviews. BMJ 2021;372:n71. doi: 10.1136/bmj.n71. For more information, visit: http://www.prisma-statement. org/ 


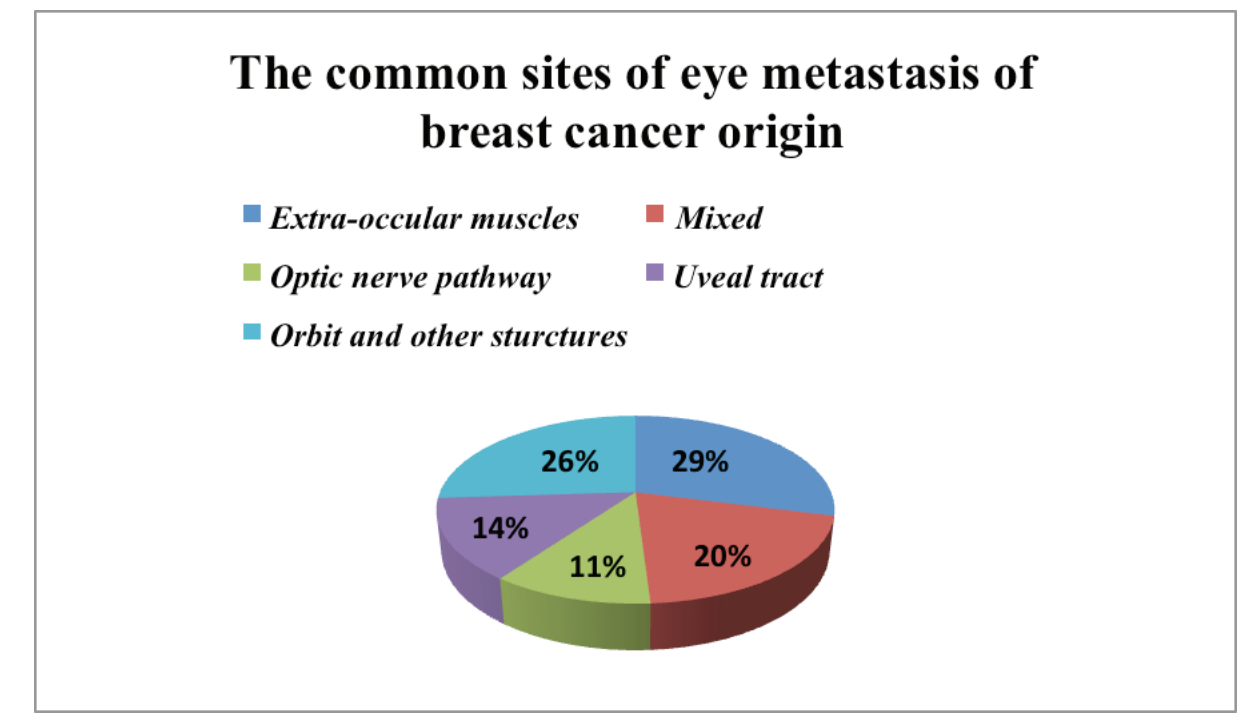

Figure 5. The most common structures within the eye with high affinity to metastasis from breast cancer.

The infiltrative ductal carcinoma (IDC) represented only half of the cases settling with one-third of patients with infiltrative lobular carcinoma (ILC); that is relatively higher than the prevalence of lobular carcinoma in the general breast cancer population [14]. The infiltrative nature of ILC could explain this discordance; Raap et al [15] reported that orbital metastases were attributed five times to ILC more often than to IDC. The luminal breast cancer subtype relates to the highest risk of eye metastasis compared with other aggressive subtypes like triple-negative breast cancer [16]. In two cases, the metastatic lesion in the eye turned ER/PR negative in primary hormone-positive breast cancer patients $[9,17]$ (Table 1).

There was a wide range of treatment modalities; mono-therapy or multimodality therapy with variable response outcomes (Table 1). Luminal breast cancer constituted the majority of cases, so we were concerned with treatment options used in hormonal positive breast cancer cases with eye metastasis. The insisting question is whether to consider eye metastasis as a visceral crisis that indicates chemotherapy or tumour progression to shift to other lines of hormonal treatment according to the guidelines [18]. Patients treated 10 years back were shifted from hormonal to chemotherapy when presented with eye metastasis resulting in a modest symptoms improvement with no available survival data $[4,5]$. Reports published in the recent 3 years [19-21] showed a clear trend towards CDK4/6 inhibitors instead of chemotherapy. This new era was associated with more local control of the disease with improvement in symptoms and considerable overall survival up to 6 years while kept on under Palbociclib [20]. The results of CDK4/6 inhibitors in the management of eye metastasis confirm the fact that the presence of eye metastasis may not jeopardise survival when treated appropriately.

Recently published cases [20, 22, 23] showed improvement in treatment outcomes secondary to the implementation of CDK4/6 inhibitors in combination with new RTH techniques like Stereotactic Body Radiotherapy (SBRT) [24]. Wiegel et al [26] showed that external beam radiotherapy (EBRTH) leads to stabilised or restored vision in up to $86 \%$ of patients; the typical dose varies between 20 and 50 Gy [3, 5, 26, 27].

The main challenges were the unavailability of CDK4/6 inhibitors or SBRT and the exhaustion of available hormonal treatment on managing rapidly progressive hormonal resistant metastatic breast cancer. That situation left chemotherapy the only reserve when presenting with eye metastasis with no response. Conformal RTH applied afterward to the persistent huge eye lesion - that was resistant to previous treatment - resulted in disease progression and symptoms worsening. 
Table 1. Summary of cases reported on the literature on eye metastasis of breast cancer origin.

\begin{tabular}{|c|c|}
\hline Number of cases & 94 (100\%) \\
\hline $\begin{array}{ll}\text { Age } & \\
\text { - Median } \\
\text { - Range }\end{array}$ & $\begin{array}{c}56 \\
(33-76)\end{array}$ \\
\hline $\begin{array}{l}\text { Histopathology } \\
\text { - IDC } \\
\text { - ILC } \\
\text { - Rare histology } \\
\text { - Unknown }\end{array}$ & $\begin{array}{c}51(54.3 \%) \\
28(29.8 \%) \\
11(11.7 \%) \\
4(4.2 \%) \\
\end{array}$ \\
\hline $\begin{array}{l}\text { Immunohistochemical subtype } \\
\text { - Hormonal receptors positive } \\
\text { - HER2 neu enriched } \\
\text { - Triple negative } \\
\text { - Not identified }\end{array}$ & $\begin{array}{c}75(80 \%) \\
9(1 \%) \\
19(2 \%) \\
16(17 \%) \\
\end{array}$ \\
\hline $\begin{array}{l}\text { Treatment modality } \\
\text {-Mono-therapy treatment: } \\
\text { Surgery alone } \\
\text { EBRTH alone } \\
\text { Hormonal alone } \\
\text { Chemotherapy alone } \\
\text { - Multimodality treatment: } \\
\text { Chemotherapy + anti-HER2 neu } \\
\text { Hormonal + RT } \\
\text { Chemotherapy + RT } \\
\text { Surgery + chemotherapy + RTH + hormonal } \\
\text { Surgery + chemotherapy+ RTH } \\
\text { Surgery + RT } \\
\text { RTH + chemotherapy +hormonal } \\
\text { Not identified or overlap of data }\end{array}$ & $\begin{array}{c}3 \text { (3.2\%) } \\
23(24.4 \%) \\
13(13.8 \%) \\
3 \text { (3.2\%) } \\
2(2.1 \%) \\
2(2.1 \%) \\
4(4.2 \%) \\
2(2.1 \%) \\
1(1.06 \%) \\
1(1.06 \%) \\
2(2.1 \%) \\
38(40.4 \%)\end{array}$ \\
\hline $\begin{array}{l}\text { Primary response } \\
\text { Partial/complete response } \\
\text { Stable disease } \\
\text { Progression } \\
\text { Not reported }\end{array}$ & $\begin{array}{c}14(14.8 \%) \\
2(2.1 \%) \\
4(4.2 \%) \\
73(77.6 \%)\end{array}$ \\
\hline
\end{tabular}

anfiltrating ductal carcinoma

bInfiltrating lobular carcinoma

'External beam radiotherapy

\section{Conclusion}

With the rising incidence of ocular metastasis due to breast cancer, oncologists should be aware of symptoms and the proper diagnostic modalities. Follow-up on the outcome of treatment is extremely crucial in the absence of guidelines that could help clinical decision. Implementation of CDK4/6 inhibitors and new techniques in RTH in the treatment of breast cancer with eye metastasis opens up new horizons for improving outcomes. We believe that reporting and sharing experiences with these cases is paramount given the relative scarcity of data in this domain. 


\section{Acknowledgment}

The authors acknowledge the patient and her family for their cooperation.

The authors thank Dr Hatem Azim Jr for his mentorship as a part of the virtual mentorship programme of the College of the European School of Oncology (ESCO).

\section{Funding sources}

This research did not receive any specific grant from funding agencies in the public, commercial or not-for-profit sectors.

\section{Conflicts of interest}

The authors have no conflicts of interest to declare.

\section{References}

1. Wickremasinghe S, Dansingani KK, and Tranos P, et al (2007) Ocular presentations of breast cancer Acta Ophthalmol Scand 85(2) 133142 https://doi.org/10.1111/j.1600-0420.2006.00737.x PMID: 17305726

2. Shaikh R, Tariq K, and Sharma S, et al (2018) Metastatic breast cancer presenting as orbital mass: a case report with literature review J Glob Oncol 4 1-4 PMID: 30241170 PMCID: 6180836

3. Ahmad SM and Esmaeli B (2007) Metastatic tumors of the orbit and ocular adnexa Curr Opin Ophthalmol 18(5) 405-413 https://doi. org/10.1097/ICU.0b013e3282c5077c PMID: 17700235

4. Vlachostergios PJ, Voutsadakis IA, and Papandreou CN (2009) Orbital metastasis of breast carcinoma Breast Cancer Basic Clin Res 3 91-97 https://doi.org/10.1177/117822340900300001

5. Eckardt AM, Rana M, and Essig H, et al (2011) Orbital metastases as first sign of metastatic spread in breast cancer: case report and review of the literature Head Neck Oncol 3(1) 1-4 https://doi.org/10.1186/1758-3284-3-37

6. Razem B and Slimani F (2021) An early orbital metastasis from breast cancer: a case report Int J Surg Case Rep 78 300-302 https://doi. org/10.1016/j.jjscr.2020.12.049 PMID: 33383286 PMCID: 7777453

7. Surace D, Piscioli I, and Morelli L, et al (2008) Orbital metastasis as the first sign of "Dormant" breast cancer dissemination 25 years after mastectomy Jpn J Ophthalmol 52(5) 423-425 https://doi.org/10.1007/s10384-008-0555-5 PMID: 18991053

8. Freedman MI and Folk JC (1987) Metastatic tumors to the eye and orbit: patient survival and clinical characteristics Arch Ophthalmol 105(9) 1215-1219 https://doi.org/10.1001/archopht.1987.01060090073031 PMID: 3307718

9. Grajales-Alvarez R and Gutierrez-Mata A (2020) Orbital metastases from breast cancer: a retrospective analysis of 28 cases Cancer Treat Res Commun 24 PMID: 32575027

10. Janicijevic-Petrovic M, Sreckovic S, and Vulovic D, et al (2011) Orbital metastases from breast cancer: a case report Basic Med Sci 11(4) 253-255

11. Ashton $\mathrm{N}$ and Morgan G (1974) Discrete carcinomatous metastases in the extraocular muscles Br J Ophthalmol 58(2) 112-117 https:// doi.org/10.1136/bjo.58.2.112 PMID: 4820982 PMCID: 1017322 
12. Demirci H, Shields CL, and Chao AN, et al (2003) Uveal metastasis from breast cancer in 264 patients Am J Ophthalmol 136(2) 264-271 https://doi.org/10.1016/S0002-9394(03)00192-2 PMID: 12888048

13. Mewis L and Young SE (1982) Breast carcinoma metastatic to the choroid. Analysis of 67 patients Ophthalmol 89(2) 147-151 https:// doi.org/10.1016/S0161-6420(82)34838-1

14. Chikman B, Lavy R, and Davidson T, et al (2010) Factors affecting rise in the incidence of infiltrating lobular carcinoma of the breast IMAJ: Israel Med Assoc J 12(11) 697-700

15. Raap M, Antonopoulos W, and Dämmrich M, et al (2015) High frequency of lobular breast cancer in distant metastases to the orbit Cancer Med 4(1) 104-111 https://doi.org/10.1002/cam4.331 PMCID: 4312124

16. Parrozzani R, Frizziero L, and Testi I, et al (2016) Intraocular metastases secondary to breast carcinoma correlates with upregulation of estrogen and progesterone receptor expression in the primary tumor Invest Ophthalmol Visual Sci 57(10) 3944-3948 https://doi. org/10.1167/iovs.16-19695

17. Wong ZW, Phillips SJ, and Ellis MJ (2004) Dramatic response of choroidal metastases from breast cancer to a combination of transtuzumab and vinorelbine Breast J 10 54-56 https://doi.org/10.1111/j.1524-4741.2004.09614.x PMID: 14717761

18. Cardoso F, Costa A, and Norton L, et al (2018) ESMO international consensus guidelines for advanced breast cancer (ABC 4) Ann Oncol 29(8) 1634-1657 https://doi.org/10.1093/annonc/mdy192 PMID: 30032243 PMCID: 7360146

19. Togashi K, Nishitsuka K, and Hayashi S, et al (2021) Metastatic orbital tumor from breast ductal carcinoma with neuroendocrine differentiation initially presenting as ocular symptoms: a case report and literature review Front Endocrinol 12 https://doi.org/10.3389/ fendo.2021.625663

20. Dupuis JEK, Marchand M, and Javidi S, et al (2021) Enophthalmos as the initial systemic finding of undiagnosed metastatic breast carcinoma Int Med Case Rep J 14 25-31 https://doi.org/10.2147/IMCRJ.S282113

21. Salinas-Botrán A and Guarín-Corredor MJ (2019) Orbital metastasis in breast cancer N Engl J Med 381(12) e26 https://doi.org/10.1056/ NEJMicm1814570 PMID: 31532964

22. Piezzo M, Chiodini P, and Riemma M, et al (2020) Progression-free survival and overall survival of CDK $4 / 6$ inhibitors plus endocrine therapy in metastatic breast cancer: a systematic review and meta-analysis Int J Mol Sci 21(17) 6400 https://doi.org/10.3390/ ijms21176400 PMCID: 7503849

23. Kim CU and Pearce WA (2020) Metastatic breast carcinoma involving the optic disc Am J Ophthalmol Case Rep 18 PMID: 32322751 PMCID: 7170934

24. Cárdenas JRV, Penella ADV, and Ibarra EC (2020) Frameless radiosurgery for intraocular metastatic tumor: case report Rep Pract Oncol Radiother 25(1) 1-5 https://doi.org/10.1016/j.rpor.2019.10.001 PMID: 32042271 PMCID: 7000959

25. Shields JA, Shields CL, and Scartozzi R (2004) Survey of 1264 patients with orbital tumors and simulating lesions: the 2002 Montgomery Lecture, part 1 Ophthalmology 111(5) 997-1008 https://doi.org/10.1016/j.ophtha.2003.01.002 PMID: 15121380

26. Wiegel T, Bottke D, and Kreusel KM, et al (2002) External beam radiotherapy of choroidal metastases - final results of a prospective study of the German Cancer Society (ARO 95-08) Radiother Oncol 64(1) 13-18 https://doi.org/10.1016/S0167-8140(02)00134-2 PMID: 12208569

27. Kadivar M, Joulaee A, and Kashkouli MB, et al (2006) Orbital Metastasis as the first presentation of nonpalpable invasive lobular carcinoma of the breast Breast J 12(1) 75-76 https://doi.org/10.1111/j.1075-122X.2006.00191.x PMID: 16409592

28. Nirmala S, Krishnaswamy M, and Janaki MG, et al (2008) Unilateral solitary choroid metastasis from breast cancer: rewarding results of external radiotherapy J Cancer Res Ther 4206 https://doi.org/10.4103/0973-1482.44295 PMID: 19052398 


\section{Supplementary material}

Supplementary Table 1. Summary of cases reported on the literature on eye metastasis of breast cancer origin.

\begin{tabular}{|c|c|c|c|c|c|c|c|c|c|c|}
\hline $\begin{array}{l}\text { Case } \\
\text { num }\end{array}$ & $\begin{array}{l}\text { Year of } \\
\text { publication }\end{array}$ & Age & Symptom & Histopathology & $\begin{array}{l}\text { Molecular } \\
\text { subtype }\end{array}$ & $\begin{array}{l}\text { Course of } \\
\text { disease }\end{array}$ & $\begin{array}{l}\text { Site of } \\
\text { metastasis }\end{array}$ & Intervention & Outcome & Ref \\
\hline 1 & 2021 & 50 & $\begin{array}{l}\text { Foreign body } \\
\text { sensation and } \\
\text { exophthalmos } \\
\text { in her right } \\
\text { eye }\end{array}$ & $\begin{array}{l}\text { IDC with } \\
\text { neuroendocrine } \\
\text { differentiation }\end{array}$ & $\begin{array}{l}\text { ER+ } \\
\text { PR + } \\
\text { Her2 - } \\
\text { KI67 high } \\
\text { Synaptophysin } \\
+\end{array}$ & $\begin{array}{l}\text { Initial } \\
\text { presentation } \\
\text { and only } \\
\text { site of } \\
\text { metastasis }\end{array}$ & $\begin{array}{l}\text { Extraoccular } \\
\text { muscles } \\
\text { and bone } \\
\text { destruction }\end{array}$ & $\begin{array}{l}3 \text { cycles nab- } \\
\text { paclitaxel followed } \\
\text { by Abemaciclib } \\
\text { plus Letrazole }\end{array}$ & $\begin{array}{l}\text { Marked } \\
\text { reduction } \\
\text { of size of } \\
\text { mass with } \\
\text { improvement } \\
\text { in visual } \\
\text { acuity. }\end{array}$ & [1] \\
\hline 2 & 2021 & 74 & $\begin{array}{l}\text { Left eye } \\
\text { enophthalmos } \\
\text { and ptosis. }\end{array}$ & IDC & $\begin{array}{l}\text { ER+ } \\
\text { PR+ } \\
\text { Her2 - }\end{array}$ & $\begin{array}{l}\text { Initial } \\
\text { presentation } \\
\text { and only } \\
\text { site of } \\
\text { metastasis }\end{array}$ & Optic nerve & $\begin{array}{l}\text { Letrazole for one } \\
\text { year then addition } \\
\text { of Palbociclib on } \\
\text { bone progression }\end{array}$ & $\begin{array}{l}\text { Control for } \\
\text { one year on } \\
\text { Letrazole } \\
\text { then kept on } \\
\text { follow-up for } \\
6 \text { years on } \\
\text { Palbociclib } \\
\text { with } \\
\text { preserved } \\
\text { visual acuity }\end{array}$ & [2] \\
\hline 3 & 2021 & 76 & $\begin{array}{l}\text { Enophthalmos } \\
\text { and ptosis. }\end{array}$ & IDC & $\begin{array}{l}\mathrm{ER}+ \\
\mathrm{PR}+ \\
\mathrm{Her} 2-\end{array}$ & $\begin{array}{l}\text { Initial } \\
\text { presentation } \\
\text { and only } \\
\text { site of } \\
\text { metastasis }\end{array}$ & $\begin{array}{l}\text { Extraoccular } \\
\text { muscles, } \\
\text { lacrimal gland } \\
\text { and the optic } \\
\text { nerve }\end{array}$ & $\begin{array}{l}\text { Letrazole and } \\
\text { Palbociclib } \\
\text { then SBRT on } \\
\text { progression }\end{array}$ & $\begin{array}{l}\text { Progression } \\
\text { on hormonal } \\
\text { treatment } \\
\text { after few } \\
\text { months with } \\
\text { OS of three } \\
\text { years. }\end{array}$ & [2] \\
\hline 4 & 2021 & 33 & $\begin{array}{l}\text { Blurring } \\
\text { vision }\end{array}$ & $\mathrm{N} / \mathrm{A}$ & $\begin{array}{l}\mathrm{ER}+ \\
\mathrm{PR}+\end{array}$ & $\begin{array}{l}\text { Previously } \\
\text { diagnosed } \\
\text { with EBC } \\
\text { one month }\end{array}$ & $\begin{array}{l}\text { Medial rectus } \\
\text { muscle and } \\
\text { the optic } \\
\text { nerve }\end{array}$ & $\begin{array}{l}\text { Treated as an EBC } \\
\text { case with surgery } \\
\text { followed by } \\
\text { adjuvant chemo- } \\
\text { radiotherapy } \\
\text { and hormonal } \\
\text { treatment }\end{array}$ & $\begin{array}{l}\text { Stable } \\
\text { disease at } \\
\text { three month } \\
\text { follow up }\end{array}$ & [3] \\
\hline 5 & 2020 & 54 & $\begin{array}{l}\text { Blurring } \\
\text { vision }\end{array}$ & IDC & $\begin{array}{l}\text { ER- } \\
\text { PR- } \\
\text { Her2 - }\end{array}$ & $\begin{array}{l}\text { Previously } \\
\text { diagnosed } \\
\text { with EBC } \\
4 \text { years } \\
\text { followed by } \\
\text { metastasis } \\
\text { to bone and } \\
\text { lung }\end{array}$ & Choroid & $\begin{array}{l}\text { Cyperknife } 18 \mathrm{GY} \\
\text { in single fraction }\end{array}$ & $\begin{array}{l}\text { Total } \\
\text { blindness } \\
\text { with partial } \\
\text { response of } \\
\text { the choroid } \\
\text { mass }\end{array}$ & [4] \\
\hline
\end{tabular}


Supplementary Table 1. Summary of cases reported on the literature on eye metastasis of breast cancer origin. (Continued)

\begin{tabular}{|c|c|c|c|c|c|c|c|c|c|c|}
\hline 6 & 2020 & $\begin{array}{l}28 \\
\text { cases } \\
\text { with } \\
\text { median } \\
\text { age } 55\end{array}$ & N/A & $\begin{array}{ll}\text { IDC } & 1 \\
\text { ILC } & \\
\text { Mixed } & 2 \\
\text { Unknown } & 3\end{array}$ & $\begin{array}{ll}21 & \text { ER+ } \\
17 & \text { PR+ } \\
20 & \text { HER2 - } \\
3 & \text { HER2+ }\end{array}$ & $\begin{array}{l}14 \text { Initial } \\
\text { presentation } \\
14 \\
\text { following } \\
\text { previous } \\
\text { diagnosis } \\
\text { with breast } \\
\text { cancer }\end{array}$ & 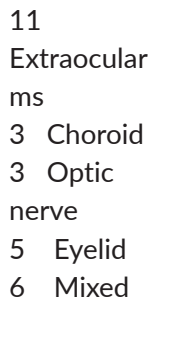 & $\begin{array}{l}14 \text { Letrazole } \\
20 \text { radiotheray } \\
\text { As first line so } \\
\text { treatment }\end{array}$ & $\begin{array}{l}\text { Median OS } \\
\text { was } 82.1 \\
\text { months }\end{array}$ & [5] \\
\hline 7 & 2020 & 46 & $\begin{array}{l}\text { Left eye } \\
\text { swelling and } \\
\text { exophthalmos }\end{array}$ & IDC & $\begin{array}{l}\text { ER + } \\
\text { PR+ } \\
\text { HER2 - }\end{array}$ & $\begin{array}{l}\text { Initial } \\
\text { presentation }\end{array}$ & $\begin{array}{l}\text { Lateral rectus } \\
\text { muscle and } \\
\text { optic nerve }\end{array}$ & N/A & N/A & [6] \\
\hline 8 & 2020 & $\begin{array}{l}28 \\
\text { cases } \\
\text { with } \\
\text { median } \\
\text { age } \\
50.8 \\
\text { years } \\
\text { old }\end{array}$ & $\begin{array}{l}18 \text { decreased } \\
\text { visual acuity } \\
16 \text { pain }\end{array}$ & $\begin{array}{ll}\text { IDC } & 14 \\
\text { ILC } & 13 \\
\text { Papillary } & 1\end{array}$ & $\begin{array}{l}26 \text { ER+ } \\
26 \mathrm{PR}+ \\
1 \quad \text { HER2+ } \\
1 \quad \text { Triple } \\
\text { positive }\end{array}$ & $\begin{array}{l}12 \text { Initial } \\
\text { presentation } \\
16 \text { with } \\
\text { previous } \\
\text { diagnosis } \\
\text { with breast } \\
\text { cancer }\end{array}$ & $\begin{array}{l}14 \text { Retina } \\
25 \text { Orbit } \\
\text { and } \\
\text { muscles } \\
14 \text { Uveal } \\
\text { tract }\end{array}$ & $\begin{array}{l}14 \text { Hormonal } \\
\text { treatment } \\
14 \text { Chemotherapy } \\
5 \text { radiotherapy } \\
3 \text { surgery } \\
1 \text { intra-ocular } \\
\text { Bevacizumab } \\
\text { As first line } \\
\text { treatment }\end{array}$ & $\begin{array}{l}\text { Median OS } \\
\text { after the } \\
\text { diagnosis } \\
\text { with eye } \\
\text { metastasis is } \\
26.4 \text { months }\end{array}$ & [7] \\
\hline 9 & 2020 & 65 & $\begin{array}{l}\text { Blurring } \\
\text { vision }\end{array}$ & IDC & $\begin{array}{l}\text { ER + } \\
\text { PR+ } \\
\text { HER2 - }\end{array}$ & $\begin{array}{l}\text { Previously } \\
\text { diagnosed } \\
\text { with EBC } 4 \\
\text { years ago }\end{array}$ & $\begin{array}{l}\text { Optic disc } \\
\text { and optic } \\
\text { nerve }\end{array}$ & SBRT & $\begin{array}{l}\text { Partial } \\
\text { response } \\
\text { improvement } \\
\text { of visual } \\
\text { acuity }\end{array}$ & [8] \\
\hline 10 & 2019 & 60 & $\begin{array}{l}\text { Blurring } \\
\text { vision, } \\
\text { proptosis }\end{array}$ & IDC & $\begin{array}{l}\text { ER + } \\
\text { PR+ } \\
\text { HER2 - }\end{array}$ & $\begin{array}{l}\text { Previously } \\
\text { diagnosed } \\
3 \text { years for } \\
\text { EBC* }^{*}\end{array}$ & $\begin{array}{l}\text { Inferior } \\
\text { rectus muscle }\end{array}$ & $\begin{array}{l}\text { Fulvestrant } \\
\text { and Palbociclib, } \\
\text { followed by } \\
\text { radiation therapy }\end{array}$ & $\begin{array}{l}\text { Follow up for } \\
6 \text { months, } \\
\text { she was free } \\
\text { clinically and } \\
\text { radiologically }\end{array}$ & [9] \\
\hline 11 & 2019 & 63 & Diplopia & IDC & $\begin{array}{l}\text { Primary } \\
\text { ER/PR+ } \\
\text { HER2- } \\
\text { Metastasis } \\
\text { Triple } \\
\text { negative } \\
\end{array}$ & $\begin{array}{l}\text { Previously } \\
\text { diagnosed } \\
\text { one year } \\
\text { before with } \\
\text { MBC }{ }^{* *}\end{array}$ & $\begin{array}{l}\text { Right medial } \\
\text { rectus muscle }\end{array}$ & $\begin{array}{l}\text { Diagnostic } \\
\text { biobsy followed } \\
\text { by palliative } \\
\text { radiotherapy and } \\
\text { chemotherapy }\end{array}$ & $\begin{array}{l}\text { Partial } \\
\text { improvement }\end{array}$ & [10] \\
\hline 12 & 2019 & 61 & $\begin{array}{l}\text { orbital } \\
\text { fullness } \\
\text { with } \\
\text { worsening } \\
\text { vision and } \\
\text { eye pain }\end{array}$ & NA & $\mathrm{ER}+$ & $\begin{array}{l}\text { Initial } \\
\text { presentation }\end{array}$ & $\begin{array}{l}\text { large ill- } \\
\text { defined } \\
\text { orbital mass } \\
\text { encasing the } \\
\text { globe }\end{array}$ & evisceration & recovered & [11] \\
\hline
\end{tabular}


Supplementary Table 1. Summary of cases reported on the literature on eye metastasis of breast cancer origin. (Continued)

\begin{tabular}{|c|c|c|c|c|c|c|c|c|c|c|}
\hline 13 & 2019 & 39 & $\begin{array}{l}\text { Decrease } \\
\text { visual acuity }\end{array}$ & IDC & $\mathrm{ER}+$ & $\begin{array}{l}\text { Previously } \\
\text { diagnosed } \\
6 \text { years ago } \\
\text { for EBC }\end{array}$ & $\begin{array}{l}\text { Mass in the } \\
\text { right optic } \\
\text { disc with } \\
\text { infiltrative } \\
\text { optic } \\
\text { neuropathy }\end{array}$ & NA & NA & [12] \\
\hline 14 & 2018 & 58 & Orbital mass & $\begin{array}{l}\text { Infiltrating } \\
\text { carcinoma }\end{array}$ & $\begin{array}{l}\text { ER + } \\
\text { PR+ } \\
\text { HER2 - }\end{array}$ & $\begin{array}{l}\text { Initial } \\
\text { presentation }\end{array}$ & $\begin{array}{l}\text { Superior } \\
\text { orbital rim }\end{array}$ & $\begin{array}{l}\text { Indoximod plus } \\
\text { Docetaxel }\end{array}$ & NA & [13] \\
\hline 15 & 2018 & 46 & Diplopia & NA & NA & $\begin{array}{l}\text { Previously } \\
\text { diagnosed } \\
\text { as MBC }\end{array}$ & $\begin{array}{l}\text { Upper left } \\
\text { oblique } \\
\text { muscle }\end{array}$ & $\begin{array}{l}\text { liposomal } \\
\text { Doxorubicin } \\
\text { as well as local } \\
\text { stereotactic } \\
\text { radiotherapy. }\end{array}$ & $\begin{array}{l}\text { showed } \\
\text { a gradual } \\
\text { improvement } \\
\text { of the local } \\
\text { symptoms } \\
\text { and signs }\end{array}$ & [14] \\
\hline 16 & 2017 & 46 & left eye pain & NA & NA & $\begin{array}{l}\text { Previously } \\
\text { diagnosed } \\
2 \text { years } \\
\text { as locally } \\
\text { advanced } \\
\text { breast } \\
\text { cancer }\end{array}$ & $\begin{array}{l}\text { Subretinal } \\
\text { mass }\end{array}$ & NA & NA & [15] \\
\hline 17 & 2017 & 56 & $\begin{array}{l}\text { Diplopia, } \\
\text { decreased } \\
\text { visual } \\
\text { acuity and } \\
\text { limited eye } \\
\text { movement }\end{array}$ & IDC & $\mathrm{ER}+$ & $\begin{array}{l}\text { Initial } \\
\text { presentation }\end{array}$ & $\begin{array}{l}\text { Intraconal } \\
\text { compartment } \\
\text { of the left } \\
\text { orbit and } \\
\text { invading } \\
\text { the adjacent } \\
\text { muscles }\end{array}$ & Excisional biobsy & NA & [16] \\
\hline 18 & 2015 & 77 & Proptosis & IDC & $\begin{array}{l}\text { ER + } \\
\text { PR - } \\
\text { HER2 - }\end{array}$ & $\begin{array}{l}\text { Previously } \\
\text { diagnosed } \\
11 \text { years } \\
\text { with EBC }\end{array}$ & $\begin{array}{l}\text { lateral and } \\
\text { superior } \\
\text { rectus } \\
\text { muscle, and } \\
\text { eroded the } \\
\text { lateral orbital } \\
\text { wall and roof }\end{array}$ & $\begin{array}{l}\text { palliative } \\
\text { radiotherapy }\end{array}$ & $\begin{array}{l}\text { Died } 9 \\
\text { months later }\end{array}$ & [17] \\
\hline 19 & 2015 & 69 & Proptosis & IDC & $\begin{array}{l}\text { ER + } \\
\text { PR - } \\
\text { HER2 - }\end{array}$ & $\begin{array}{l}\text { Previously } \\
\text { diagnosed } \\
\text { one year for } \\
\text { EBC }\end{array}$ & $\begin{array}{l}\text { Lacrimal } \\
\text { gland }\end{array}$ & $\begin{array}{l}\text { palliative } \\
\text { radiotherapy } \\
\text { and one cycle } \\
\text { chemotherapy }\end{array}$ & $\begin{array}{l}\text { Died } 3 \\
\text { month later }\end{array}$ & [17] \\
\hline 20 & 2019 & 56 & $\begin{array}{l}\text { Decreased } \\
\text { visual acuity }\end{array}$ & IDC & NA & $\begin{array}{l}\text { Initial } \\
\text { presentation }\end{array}$ & Choridal & NA & NA & [18] \\
\hline 21 & 2014 & 84 & Diplopia & NA & NA & $\begin{array}{l}\text { Previously } \\
\text { diagnosed } \\
14 \text { years } \\
\text { EBC }\end{array}$ & Orbit & NA & NA & [19] \\
\hline
\end{tabular}


Supplementary Table 1. Summary of cases reported on the literature on eye metastasis of breast cancer origin. (Continued)

\begin{tabular}{|c|c|c|c|c|c|c|c|c|c|c|}
\hline 22 & 2012 & 48 & $\begin{array}{l}\text { Blurring } \\
\text { vision }\end{array}$ & IDC & NA & $\begin{array}{l}\text { Previously } \\
\text { diagnosed } \\
13 \text { years } \\
\text { EBC }\end{array}$ & $\begin{array}{l}\text { Choroidal } \\
\text { mass }\end{array}$ & $\begin{array}{l}\text { Surgical } \\
\text { enucleation }\end{array}$ & NA & [20] \\
\hline 23 & 2012 & 53 & $\begin{array}{l}\text { Proptosis and } \\
\text { diplopia }\end{array}$ & NA & NA & $\begin{array}{l}\text { Initial } \\
\text { presentation }\end{array}$ & $\begin{array}{l}\text { Right orbit } \\
\text { and skull } \\
\text { base }\end{array}$ & $\begin{array}{l}\text { Surgery and } \\
\text { palliative } \\
\text { radiotherapy }\end{array}$ & NA & [21] \\
\hline 24 & 2011 & 73 & Exophthalmus & IDC & $\begin{array}{l}\mathrm{ER}+ \\
\mathrm{PR}+ \\
\mathrm{HER} 2+\end{array}$ & $\begin{array}{l}\text { Previous } \\
\text { diagnosed } 3 \\
\text { ys before as } \\
\text { EBC }\end{array}$ & $\begin{array}{l}\text { Suprabulbar } \\
\text { tumor mass, } \\
\text { with } \\
\text { osseous } \\
\text { infiltration } \\
\text { of the orbital } \\
\text { roof }\end{array}$ & $\begin{array}{l}\text { Palliative } \\
\text { chemotherapy } \\
\text { plus } \\
\text { bisphosphonates }\end{array}$ & $\begin{array}{l}\text { Improvement } \\
\text { in symptom } \\
\text { and continue } \\
\text { chemo then } \\
\text { hormonal } \\
\text { treatment }\end{array}$ & [22] \\
\hline 25 & 2011 & 38 & $\begin{array}{l}\text { Visual } \\
\text { discomfort }\end{array}$ & NA & NA & $\begin{array}{l}\text { Previously } \\
\text { diagnosed } 3 \\
\text { years with } \\
\text { MBC }\end{array}$ & $\begin{array}{l}\text { Bilateral } \\
\text { orbital } \\
\text { involvement }\end{array}$ & SPRT & $\begin{array}{l}\text { Corrected } \\
\text { visual acuity } \\
\text { but died } 19 \\
\text { month later }\end{array}$ & [23] \\
\hline 26 & 2010 & 66 & $\begin{array}{l}\text { Swelling } \\
\text { and visual } \\
\text { disorders }\end{array}$ & ILC & $\begin{array}{l}\mathrm{ER}+ \\
\mathrm{PR}+ \\
\text { HER2 - }\end{array}$ & $\begin{array}{l}\text { Initial } \\
\text { presentation }\end{array}$ & $\begin{array}{l}\text { Right peri- } \\
\text { orbital soft } \\
\text { tissues }\end{array}$ & $\begin{array}{l}\text { Surgery for } \\
\text { primary and } \\
\text { metastasis then } \\
\text { FEC and palliative } \\
\text { radiotherapy } \\
\text { followed by } \\
\text { hormonal } \\
\text { treatment }\end{array}$ & $\begin{array}{l}\text { Total } \\
\text { resolution }\end{array}$ & [24] \\
\hline 27 & 2009 & 46 & $\begin{array}{l}\text { Diplopia, } \\
\text { proptosis }\end{array}$ & IDC & $\begin{array}{l}\mathrm{ER}+ \\
\mathrm{PR}+ \\
\text { HER2 - }\end{array}$ & $\begin{array}{l}\text { Previously } \\
\text { diagnosed } 4 \\
\text { years ago as } \\
\text { MBC }\end{array}$ & $\begin{array}{l}\text { Intra-orbital } \\
\text { extra-bulbar } \\
\text { mass }\end{array}$ & $\begin{array}{l}\text { Chemotherapy } \\
\text { plus Cyperknife }\end{array}$ & $\begin{array}{l}\text { Gradual } \\
\text { improvement } \\
\text { in symptoms } \\
\text { Free of } \\
\text { ocular for } 18 \\
\text { months }\end{array}$ & [25] \\
\hline 28 & 2009 & 70 & $\begin{array}{l}\text { Dimension of } \\
\text { vision }\end{array}$ & NA & NA & $\begin{array}{l}\text { Previously } \\
\text { diagnosed } 9 \\
\text { ys early EBC }\end{array}$ & Choroid & NA & NA & [26] \\
\hline 29 & 2008 & 73 & $\begin{array}{l}\text { Ptosis and } \\
\text { diplopia }\end{array}$ & ILC & $\begin{array}{l}\mathrm{ER}+ \\
\mathrm{PR}+\end{array}$ & $\begin{array}{l}\text { Previously } \\
\text { diagnosed } \\
25 \text { years ago } \\
\text { for EBC }\end{array}$ & $\begin{array}{l}\text { Extrinsic } \\
\text { muscles } \\
\text { and the } \\
\text { surrounding } \\
\text { tissues }\end{array}$ & NA & NA & [27] \\
\hline 30 & 2008 & 50 & Diplopia & ILC & $\begin{array}{l}\mathrm{ER}+ \\
\mathrm{PR}+\end{array}$ & $\begin{array}{l}\text { Previously } \\
\text { diagnosed } \\
5 \text { years for } \\
\text { stage III }\end{array}$ & $\begin{array}{l}\text { Bilateral } \\
\text { extra-ocular } \\
\text { muscles }\end{array}$ & $\begin{array}{l}\text { High dose } \\
\text { radiotherapy, } \\
\text { hormonal and } \\
\text { chemotherapy }\end{array}$ & NA & [28] \\
\hline
\end{tabular}


Supplementary Table 1. Summary of cases reported on the literature on eye metastasis of breast cancer origin. (Continued)

\begin{tabular}{|c|c|c|c|c|c|c|c|c|c|c|}
\hline 31 & 2008 & 66 & $\begin{array}{l}\text { Unilateral } \\
\text { eyelid edema }\end{array}$ & NA & $\begin{array}{l}\text { ER + } \\
\text { PR+ } \\
\text { HER2 + }\end{array}$ & $\begin{array}{l}\text { Previously } \\
\text { diagnosed } \\
\text { with stage III }\end{array}$ & $\begin{array}{l}\text { Soft tissue } \\
\text { mass in orbit }\end{array}$ & $\begin{array}{l}\text { Trastuzumab, } \\
\text { Docetaxel, } \\
\text { Tegafur and } \\
\text { Cyclophosphamide }\end{array}$ & $\begin{array}{l}\text { Complete } \\
\text { response }\end{array}$ & [29] \\
\hline 32 & 2008 & 60 & $\begin{array}{l}\text { Diminution of } \\
\text { vision }\end{array}$ & IDC & $\begin{array}{l}\mathrm{ER}+ \\
\mathrm{PR}+\end{array}$ & $\begin{array}{l}\text { Previously } \\
\text { diagnosed } 3 \\
\text { ys for EBC }\end{array}$ & $\begin{array}{l}\text { Uveal tract } \\
\text { of the right } \\
\text { globe and } \\
\text { optic disc }\end{array}$ & $\begin{array}{l}\text { Radiotherapy } 44 \\
\text { GY }\end{array}$ & $\begin{array}{l}\text { Complete } \\
\text { response }\end{array}$ & [30] \\
\hline 33 & 2006 & 53 & $\begin{array}{l}\text { Orbital pain } \\
\text { and ptosis }\end{array}$ & $I C L$ & NA & $\begin{array}{l}\text { Initial } \\
\text { presentation }\end{array}$ & $\begin{array}{l}\text { The medial } \\
\text { wall of the } \\
\text { left } \\
\text { orbit, orbital } \\
\text { connective } \\
\text { tissue, } \\
\text { muscles, and } \\
\text { lacrimal gland }\end{array}$ & NA & NA & [31] \\
\hline 34 & 2006 & 58 & $\begin{array}{l}\text { Swelling in } \\
\text { lower eye lid }\end{array}$ & ILC & $\begin{array}{l}\mathrm{ER}+ \\
\mathrm{PR}+\end{array}$ & $\begin{array}{l}\text { EBC } 24 \\
\text { month } \\
\text { before }\end{array}$ & $\begin{array}{l}\text { Mass in } \\
\text { lower eye lid }\end{array}$ & NA & NA & [32] \\
\hline 35 & 2005 & 75 & $\begin{array}{l}\text { Binocular } \\
\text { diplopia }\end{array}$ & NA & NA & $\begin{array}{l}\text { Previously } \\
\text { diagnosed } 2 \\
\text { months EBC }\end{array}$ & $\begin{array}{l}\text { Extraocular } \\
\text { muscles } \\
\text { bilaterally }\end{array}$ & NA & NA & [33] \\
\hline 36 & 2005 & 52 & $\begin{array}{l}\text { Progressive } \\
\text { visual loss } \\
\text { and ptosis }\end{array}$ & IDC & NA & $\begin{array}{l}\text { Previously } \\
\text { diagnosed } \\
\text { as MBC }\end{array}$ & $\begin{array}{l}\text { Bilateral } \\
\text { thickening } \\
\text { of all } \\
\text { extraocular } \\
\text { muscle }\end{array}$ & NA & NA & [34] \\
\hline 37 & 2004 & 35 & $\begin{array}{l}\text { Diplopia and } \\
\text { blurred vision }\end{array}$ & ILC & NA & $\begin{array}{l}\text { Previously } \\
\text { diagnosed } \\
3 \text { years ago } \\
\text { for EBC } \\
\end{array}$ & $\begin{array}{l}\text { Right lateral } \\
\text { rectus muscle }\end{array}$ & $\begin{array}{l}\text { Palliative } \\
\text { chemotherapy }\end{array}$ & $\begin{array}{l}\text { Died after } 10 \\
\text { days }\end{array}$ & [35] \\
\hline 38 & 2004 & 57 & $\begin{array}{l}\text { Decrease in } \\
\text { visual acuity }\end{array}$ & IDC & $\begin{array}{l}\text { Primary ER/ } \\
\text { PR+ } \\
\text { Metastasis } \\
\text { HER2 +, ER/ } \\
\text { PR - }\end{array}$ & $\begin{array}{l}\text { Previously } \\
\text { diagnosed } \\
13 \text { ys with } \\
\text { EBC }\end{array}$ & $\begin{array}{l}\text { Unilateral } \\
\text { choroidal } \\
\text { metastasis }\end{array}$ & $\begin{array}{l}\text { Trastuzumab plus } \\
\text { Vinolerabin }\end{array}$ & $\begin{array}{l}\text { Complete } \\
\text { response }\end{array}$ & [36] \\
\hline 39 & 2002 & 61 & $\begin{array}{l}\text { Red eye and } \\
\text { ptosis }\end{array}$ & IDC & $\mathrm{ER}+$ & $\begin{array}{l}\text { Initial } \\
\text { presentation }\end{array}$ & $\begin{array}{l}\text { Mass filling } \\
\text { the left orbit } \\
\text { posteriorly } \\
\text { and } \\
\text { extending } \\
\text { forward in } \\
\text { the eyelids } \\
\end{array}$ & $\begin{array}{l}\text { Surgery and local } \\
\text { radiotherapy } 3000 \\
\text { cGy }\end{array}$ & NA & [37] \\
\hline 40 & 2001 & 40 & Loss of vision & NA & NA & $\begin{array}{l}\text { Previously } \\
\text { diagnosed } \\
\text { EBC }\end{array}$ & $\begin{array}{l}\text { Isolated } \\
\text { choroid } \\
\text { metastasis }\end{array}$ & $\begin{array}{l}\text { Radiotherapy } \\
\text { palliative, } \\
\text { chemotherapy } \\
\text { plus acetazolatime }\end{array}$ & $\begin{array}{l}\text { Clinical and } \\
\text { radiological } \\
\text { remission }\end{array}$ & [38] \\
\hline
\end{tabular}




\section{References}

1. Togashi K, Nishitsuka K, and Hayashi S, et al (2021) Metastatic orbital tumor from breast ductal carcinoma with neuroendocrine differentiation initially presenting as ocular symptoms: a case report and literature review Front Endocrinol 202112

2. Dupuis JEK, Marchand M, and Javidi S, et al (2021) Enophthalmos as the initial systemic finding of undiagnosed metastatic breast carcinoma Int Med Case Rep J 14 25-31

3. Razem B and Slimani F (2021) An early orbital metastasis from breast cancer: a case report Int J Surg Case Rep 78 300-302

4. Cárdenas JRV, Penella ADV, and Ibarra EC (2020) Frameless radiosurgery for intraocular metastatic tumor: case report Rep Pract Oncol Radiother 25(1) 1-5

5. Blohmer M, Zhu L, and Atkinson JM, et al (2020) Patient treatment and outcome after breast cancer orbital and periorbital metastases: a comprehensive case series including analysis of lobular versus ductal tumor histology Breast Cancer Res 22(1) 70

6. Chudasama R, Hsu A, and Constantinou M (2020) Orbital metastasis in inflammatory breast cancer Breast J 26(8) 1629-1630

7. Grajales-Alvarez R and Gutierrez-Mata A (2020) Orbital metastases from breast cancer: a retrospective analysis of 28 cases Cancer Treat Res Commun 24

8. Kim CU and Pearce WA (2020) Metastatic breast carcinoma involving the optic disc Am J Ophthalmol Case Rep 18

9. Salinas-Botrán A and Guarín-Corredor MJ (2019) Orbital metastasis in breast cancer N Engl J Med 381(12) e26

10. Kızıloğlu ÖY, Türköz FP, and Gedar ÖMT, et al (2019) Breast carcinoma metastasis to the medial rectus muscle: case report Turk J Ophthalmol 49(3) 168-170

11. Danek DJ, Blessing NW, and Tse DT (2019) Corneal perforation with uveal prolapse: an initial presentation of orbital metastatic breast cancer Am J Ophthalmol Case Rep 16

12. Aghdam KA, Zand A, and Sanjari MS (2019) Isolated unilateral infiltrative optic neuropathy in a patient with breast cancer Turk J Ophthalmol 49(3) 171-174

13. Shaikh R, Tariq K, and Sharma S, et al (2018) Metastatic breast cancer presenting as orbital mass: a case report with literature review J Global Oncol 2018(4) 1-4

14. Nifosí G and Zuccarello M (2018) Unilateral localized extraocular muscle metastasis by lobular breast carcinoma BMJ Case Rep 2018 bcr-2018-224726

15. Shah RK and Lamichhane S (2017) Ocular metastasis from breast carcinoma simulating anterior scleritis: a case report J Med Case Rep 11(1) 249

16. Mărginean A, et al (2017) Intraorbital tumor as the first and sole manifestation of breast cancer Ginecoeu 13(3) 109-110

17. Nickelsen MN, von Holstein SL, and Hansen AB, et al (2015) Breast carcinoma metastasis to the lacrimal gland: two case reports Oncol lett 10(2) 1031-1035

18. Belabbes SB and Belkhadir K (2019) Ocular metastasis revealing ductal carcinoma of the breast Pan Afr Med J 3229

19. Rossi L, Zancla S, and Civitelli L, et al (2014) An unusual orbital metastasis of breast cancer Breast Dis 34(4) 173-176

20. Liu T, Xu Y, and Wan L, et al (2012) Choroid as the first recurrence site: 13 years after breast carcinoma J Cancer Res Ther 8(4) 639-640

21. Dmuchowska DA, Krasnicki P, and Obuchowska I, et al (2012) Ophthalmic manifestation of skull base metastasis from breast cancer Med Sci Monit 18(11) CS105-CS8 
22. Eckardt AM, Rana M, and Essig H, et al (2011) Orbital metastases as first sign of metastatic spread in breast cancer: case report and review of the literature Head Neck Oncol 3(1)

23. Kim JH, Choi SY, and Cho CK, et al (2011) Bilateral orbital metastases from breast cancer: a case report of successful palliation using stereotactic radiotherapy Breast J 17(6) 669-671

24. Francone E, Murelli F, and Paroldi A, et al (2010) Orbital swelling as a first symptom in breast carcinoma diagnosis: a case report J Med Case Rep 4

25. Vlachostergios PJ, Voutsadakis IA, and Papandreou CN (2009) Orbital metastasis of breast carcinoma Breast Cancer (Auckl) 391 -97

26. Solav S, Bhandari R, and Sowani A, et al (2010) Choroidal metastasis from carcinoma of breast detected on F18-FDG PET CT scan: a case report and review of literature Indian J Nucl Med 25(4) 160-163

27. Surace D, Piscioli I, and Morelli L, et al (2008) Orbital metastasis as the first sign of "Dormant" breast cancer dissemination 25 years after mastectomy Jpn J Ophthalmol 52(5) 423-425

28. Kouvaris JR, Gkongkou PV, and Papadimitriou CA, et al (2008) Bilateral metastases to extraocular muscles from lobular breast carcinoma Onkologie 31 387-389

29. Kouvaris JR, Gkongkou PV, and Papadimitriou CA, et al (2008) Bilateral metastases to extraocular muscles from lobular breast carcinoma Oncol Res Treat 31(7) 387-389

30. Nirmala S, Krishnaswamy M, and Janaki MG, et al (2008) Unilateral solitary choroid metastasis from breast cancer: rewarding results of external radiotherapy J Cancer Res Ther 4206

31. Kadivar M, Joulaee A, and Kashkouli MB, et al (2006) Orbital metastasis as the first presentation of nonpalpable invasive lobular carcinoma of the breast Breast J 12(1) 75-76

32. Kuchel JM and Bowling JC (2006) Bilateral lower eyelid masses. Orbital (intra-orbital and extraocular) metastases in breast cancer Arch Dermatol 142 1351-1356

33. Spitzer SG, Bersani TA, and Mejico LJ (2005) Multiple bilateral extraocular muscle metastases as the initial manifestation of breast cancer J Neuro Ophthalmol 25(1) 37-39

34. Peckham EL, Giblen G, and Kim AK, et al (2005) Bilateral extraocular muscle metastasis from primary breast cancer Neurology 65(1) 74

35. Asproudis I, Gorezis S, and Charalabopoulos K, et al (2004) Breast carcinoma metastasis to the orbit and paranasal sinuses: a case report Exp Oncol 26 246-248

36. Wong ZW, Phillips SJ, and Ellis MJ (2004) Dramatic response of choroidal metastases from breast cancer to a combination of transtuzumab and vinorelbine Breast J 10 54-56

37. Reeves D, Levine MR, and Lash R (2002) Nonpalpable breast carcinoma presenting as orbital infiltration: case presentation and literature review Ophthalmic Plast Reconstr Surg 18(1) 84-88

38. Sari R, Camci C, and Kutlu R, et al (2001) The efficacy of acetazolamide on visual functions in a patient with choroid metastasis of breast carcinoma Int J Clin Pract 55 488-490 